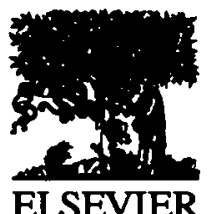

Molecular and Biochemical Parasitology 67 (1994) 183-191

MOLECULAR

\title{
Effects of antimalarials and protease inhibitors on plasmodial hemozoin production
}

\author{
Wanida Asawamahasakda ${ }^{1}$, Isra Ittarat, Chih-Ching Chang, Peter McElroy, \\ Steven R. Meshnick *
}

Department of Epidemiology, University of Michigan School of Public Health, 109 Observatory Street, Ann Arbor, MI 48109 USA

Received 8 April 1994; accepted 20 June 1994

\begin{abstract}
Malarial hemozoin may play an important role as a target for antimalarial drugs and in disease pathogenesis. A new assay for hemozoin was developed in which the hemozoin was separated from cells by filtration. Trophozoites have substantially more hemozoin than rings, but there are relatively small differences between chloroquine-sensitive and chloroquine-resistant strains. The effects on hemozoin content of chloroquine and artemisinin, two antimalarial drugs, and E64 and Pepstatin A, two protease inhibitors, were measured. At concentrations at which hypoxanthine incorporation was unaffected, the hemozoin content of rings was decreased by E64, but not by the other three compounds. Artemisinin and Pepstatin A also had little effect on the hemozoin content of trophozoites. Chloroquine and E64 inhibited trophozoite hemozoin formation, but inhibited hypoxanthine uptake to a similar or greater extent. When either rings or trophozoites were exposed to several higher concentrations of chloroquine, hemozoin content was diminished, but significantly less than hypoxanthine uptake. Various concentrations of E64, in contrast, inhibited hemozoin production by both rings and trophozoites significantly more than hypoxanthine incorporation, suggesting that hemozoin production may be directly affected by E64.
\end{abstract}

Keywords: Artemisinin; Chloroquine; E64; Haem; Malaria; Pepstatin A; Plasmodium falciparum; Qinghaosu

\section{Introduction}

Hemozoin, or malaria pigment, is formed from heme molecules that are liberated by hemoglobin degradation (reviewed in [1] and [2]). Malaria parasites produce large quantities of hemozoin since they

\footnotetext{
* Corresponding author. Tel: (313) 747 2406; Fax: (313) 764 3192; E-mail: Meshnick@umich.edu

'Present address: Department of Clinical Microscopy, Faculty of Medical Technology, Mahidol University, Siriraj Hospital, Bangkok, Thailand.
}

degrade approximately $25 \%$ of their host erythrocytes' hemoglobin [3]. Hemoglobin digestion, which occurs in the parasite food vacuole, may require both aspartic [4] and cysteine [5] proteases. Released heme is incorporated into hemozoin by the newly discovered enzyme, heme polymerase [6].

Hemozoin may play an important role in the pathogenesis of malaria. During the course of a malaria infection, hemozoin is taken up by the reticuloendothelial system. Hemozoin uptake diminishes the phagocytic capacity of macrophages, perhaps as a consequence of $\mathrm{H}_{2} \mathrm{O}_{2}$-mediated release of iron 
from hemozoin, and this may cause immunosuppression $[7,8]$.

Hemozoin may also play an important role in the modes of action of chloroquine and artemisinin. The synthesis of hemozoin is inhibited by chloroquine in cell-free extracts [6], and in both Plasmodium falciparum [9] and Plasmodium berghei [10,11]. Hemozoin has also been shown to play an important role in the mode of action of artemisinin (qinghaosu) [12-18]. Intraparasitic heme catalyzes the breakdown of artemisinin into an organic free radical [13-15] which can alkylate both proteins $[16,17]$ and heme molecules [18].

In this study, the effects of chloroquine and artemisinin on hemozoin production were measured in order to gain insight into the modes of action of these drugs. In addition, we have studied the effects of $E$ 64, a cysteine protease inhibitor, and Pepstatin $A$, and aspartic protease inhibitor, on hemozoin production in order to better understand the relative importance of the two classes of proteases.

\section{Materials and methods}

\subsection{Chemicals and supplies}

Human serum and red cells were purchased from Interstate Blood Bank (Memphis, TN). Tritiated hypoxanthine (1-5 $\left.\mathrm{Ci} \mathrm{mmol}{ }^{-1}\right)$ was purchased from Amersham Corp. (Arlington Heights, IL). RPMI 1640 was purchased from Gibco/BRL (Grand Island, NY). All other chemicals were purchased from Sigma Chemical Co. (St Louis, MO) unless otherwise noted.

\subsection{Parasites}

P. falciparum strains D6 (chloroquine-sensitive) and W2 (chloroquine-resistant) were kindly provided by $\mathrm{R}$. Miller, Walter Reed Army Institute of Research, Washington DC. P. falciparum strain FCR3 was the gift of M. Perkins, Rockefeller University, New York. Parasitized red cells were cultivated by to the method of Trager and Jensen [19]. Synchronization by sorbitol lysis [20] was performed for at least 3 consecutive cycles before parasites were used. Every synchronization was followed by careful removal of cell and hemozoin debris which is the brown-black layer at the top of the cell suspension after centrifugation. Cultures were used only if they contained $95-98 \%$ of either mature trophozoites $(26-32 \mathrm{~h})$ or small rings $(12-18 \mathrm{~h})$, the stages defined as described by Silamut and White [21]. Host cell-free parasites were obtained by saponin lysis [22]. Parasitemia and parasite morphology were determined microscopically from Giemsa-stained thin blood films, counting at least 500 erythrocytes.

\subsection{Determination of hemozoin content}

Parasite hemozoin content was assayed as follows. Isolated parasites were washed 4 times in 20 volumes of a Triton X-100 solution ( $0.05 \%$ in water), centrifuging in a microfuge at $14000 \times g$ for $5 \mathrm{~min}$ each time. The final pellet was resuspended in $1 \mathrm{ml}$ of $0.05 \%$ Triton $\mathrm{X}-100$, and placed in a Branson sonicating water bath for $10-20 \mathrm{~s}$ in order to fully suspend the pellet. The suspension was then taken up in a 5-ml syringe and passed through a 25-mm diameter 0.45-micron Metricel membrane filter (Gelman Sciences Inc., Ann Arbor Ml) and washed by passing $1 \mathrm{ml}$ of $0.05 \%$ Triton $\mathrm{X}-100$ through the filter. Retained hemozoin was assayed by a modification of the pyridine hemochromogen assay [23]. Heme was solubilized by placing the filter in $3 \mathrm{ml}$ of a reaction mixture containing $1 \mathrm{~N} \mathrm{KOH} /$ pyridine/ distilled water (1:2:8) and vortexing for $1-2 \mathrm{~min} .1$ $\mathrm{ml}$ of this solution was put into each of 2 cuvettes. A few crystals of sodium dithionite were added to one cuvette and $10 \mu \mathrm{l}$ of $50 \mathrm{mM}$ potassium ferricyanide to the other, and heme was quantitated by the difference in $A_{560}$ as measured on a Milton Roy Spectronic 2000 spectrophotometer using reagent hemin as a standard.

\subsection{Validation of assay}

To test the validity of this assay, known quantities of unpolymerized heme and synthetic polymerized $\beta$-hematin were assayed. Unpolymerized heme (10 $\mathrm{mM}$ in $0.02 \mathrm{~N} \mathrm{NaOH} /$ ethanol, 1:1) [24] and polymerized $\beta$-hematin [25] were prepared and filtered. The heme content of the retentate was assayed as above. The filtrate was concentrated on a Speedvac (Savant, Farmingdale NY) and then assayed for heme by pyridine hemochromogen [23]. 
As a second test of the validity of the assay, the hemozoin content of identical aliquots of isolated parasites were assayed simultaneously by both the method described above and the method of Slater et al. [25].

As a third test for validity, we determined whether the hemozoin content of samples was proportional to parasitemia. To do this, a suspension of trophozoiteinfected erythrocytes (10\% parasitemia, $50 \%$ hematocrit) was 2 -fold serially diluted with a suspension of uninfected erythrocytes (50\% hematocrit). $200 \mu \mathrm{l}$ of each parasite suspension was then lysed by saponin and assayed for hemozoin content as described above.

As a fourth test for validity, isolated parasite suspensions were divided in half and filtered on two identical filters. The hemozoin content of one was assayed as above. The hemoglobin content of the duplicate filter was assayed [26], and expressed as a molar percentage of the hemozoin heme content.

\subsection{The effects of inhibitors on hemozoin content and hypoxanthine incorporation}

To study the effects of antimalarials and protease inhibitors on hemozoin synthesis, $10-\mathrm{ml}$ cultures of $P$. falciparum $\left(\mathrm{D}_{6}\right.$ strain), at either mature trophozoite or small ring stage, were incubated in the presence of $0.5 \mu \mathrm{M}$ chloroquine, $0.5 \mu \mathrm{M}$ artemisinin, $50 \mu \mathrm{M} \mathrm{E} 64,50 \mu \mathrm{M}$ of pepstatin $\mathrm{A}$ or no addition. Immediately after addition of the drugs, $0.2 \mathrm{ml}$ of each treated culture was transferred to a well in a microtiter plate to which $0.5 \mu \mathrm{Ci}$ of $\left[{ }^{3} \mathrm{H}\right]$ hypoxanthine was then added. Hypoxanthine uptake was used to monitor nucleic acid biosynthesis as a general indicator of parasite viability $[27,28]$. The cultures and microtiter plates were incubated in a candle jar for $6 \mathrm{~h}$ at $37^{\circ} \mathrm{C}$. The hemozoin content of the culture plate was determined as described above. The microtiter plate was harvested using a Brandel Cell Harvester Model M24 (Brandel, Gaithersburg, MD). Filters were counted on a Beckman LS 7000 Scintillation counter (Beckman Instrument, Fullerton, CA) using Scinti-Verse BD (Fisher Scientific, Fair Lawn, $\mathrm{NJ}$ ) as scintillant. Infected erythrocytes from the same cultures incubated in the absence of inhibitors were used as controls. Concentration-response experiments were also undertaken for chloroquine and E64. $P$. falciparum $\left(\mathrm{D}_{6}\right.$ strain) was incubated in the
Table 1

Distribution of heme after filtration

\begin{tabular}{lcc}
\hline & Unpolymerized & $\beta$-hematin \\
heme & \\
\hline Retentate (nmol) & $13.0 \pm 0.4$ & $390.3 \pm 19$ \\
Filtrate (nmol) & $978 \pm 42$ & $14.5 \pm 4.1$ \\
Percent retained on filter paper & 1.3 & 96.5 \\
\hline
\end{tabular}

Data shown are means \pm S.D. of triplicate experiments.

presence of various concentrations of either inhibitor. Trophozoite-infected cells were treated exactly as above, assaying for both hemozoin content and hypoxanthine incorporation. In order to increase sensitivity, the ring-infected erythrocytes used for the concentration-response curve were incubated as above, but for $24 \mathrm{~h}$ instead of $6 \mathrm{~h}$. Statistical analyses of results were performed using two-tailed unpaired $t$-tests (Statview II, Abacus Concepts, Mountainview, CA).

\section{Results}

\subsection{Validation of assay}

The filter retains almost no unpolymerized heme but nearly all of the synthetic $\beta$-hematin. As shown in Table $1,96 \%$ of synthetic $\beta$-hematin was captured on the filter paper, while only $1.3 \%$ of unpolymerized heme was retained. Thus, polymerized heme, but not monomeric heme, is trapped on the filter, while monomeric heme, not polymerized heme, can be found in the filtrate.

The filtration assay is effective for measuring hemozoin in $\boldsymbol{P}$. falciparum-infected erythrocytes. When isolated parasite suspensions were filtered and assayed in parallel by both pyridine hemochromogen and Drabkin's reagent, only $1.52 \pm 0.7 \%(N=4)$ of the retained heme was found to be hemoglobinbound. Furthermore, as expected, the hemozoin content was found to vary linearly with parasitemia (Fig. $1 ; r^{2}=0.99$ ).

The filtration method described here agrees well with the method of Slater et al. [25]. When different preparations of isolated parasites were assayed by both methods, and the two methods were plotted against each other, there was a very strong correla- 


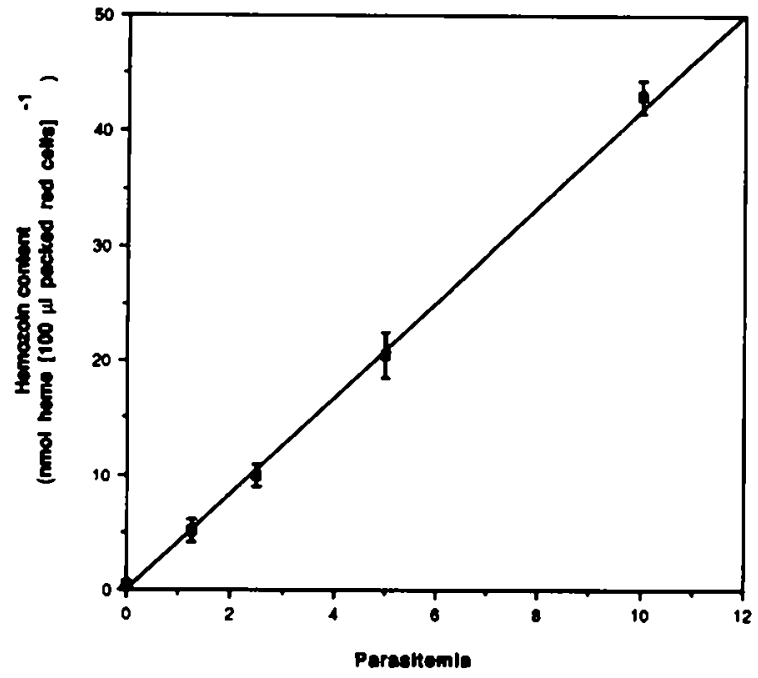

Fig. 1. Correlation between parasitemia and hemozoin content in trophozoite-infected erythrocytes. Error bars represent standard deviations of triplicate experiments.

tion (Fig. 2), with a slope of 0.999 and an $r^{2}$ of 0.998 .

\subsection{Hemozoin content in chloroquine-sensitive and chloroquine-resistant strains of $P$. falciparum}

A marked difference was found in the hemozoin content of trophozoites and rings (Table 2). Tropho-

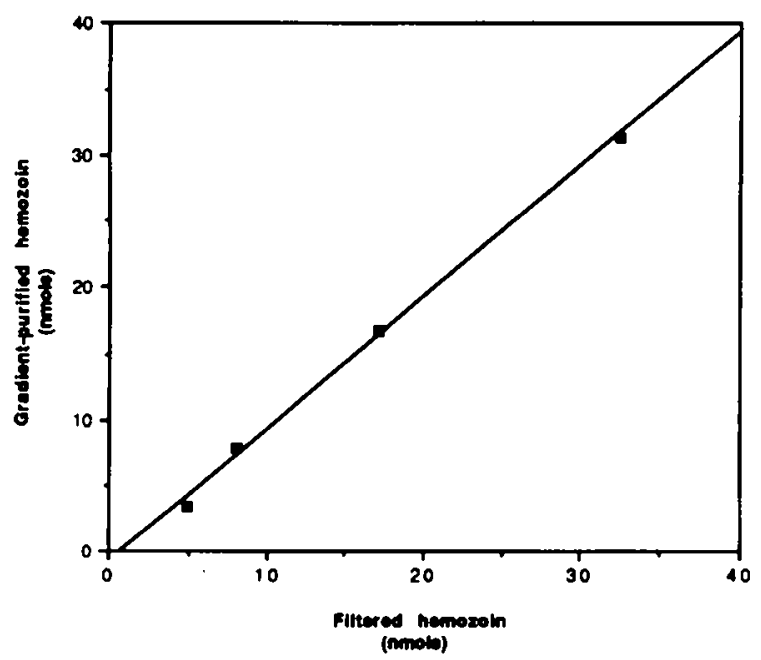

Fig. 2. Hemozoin content at various parasitemias measured by the method of Slater et al. [25] (y-axis) and the filtration method described here ( $\mathrm{x}$-axis).
Table 2

Hemozoin contents of infected and uninfected red cells

\begin{tabular}{lc}
\hline Cell type & pmol $\left(10^{6}\right.$ cells) ${ }^{-1}$ \\
\hline Uninfected red cells & $1.0 \pm 0.3$ \\
$\begin{array}{l}\text { Chloroquine-sensitive }\left(D_{6}\right)- \\
\text { ring stage }\end{array}$ & $74.6 \pm 5.7$ \\
$\begin{array}{l}\text { Chloroquine-sensitive ( } \\
\text { trophozoite stage }\end{array}$ & $637 \pm 91$ \\
$\begin{array}{l}\text { Chloroquine-resistant (FCR 3)- } \\
\text { trophozoite stage }\end{array}$ & $781 \pm 25$ \\
$\begin{array}{l}\text { Chloroquine-resistant (W 2)- } \\
\text { trophozoite stage }\end{array}$ & $952 \pm 78$ \\
\hline
\end{tabular}

Data shown are means \pm S.D. of triplicate experiments.

zoites of $D_{6}$ strain contain $637 \pm 91$ pmol of hemozoin-heme $\left(10^{6} \text { parasitized cells }\right)^{-1}$, eight times more than rings, which contain only $74.6 \pm 9.7 \mathrm{pmol}\left(10^{6}\right.$ parasitized cells) $)^{-1}$. In contrast, negligible amounts of hemozoin were found in uninfected red cells $\left(1.0 \pm 0.3\right.$ pmol $\left.\left(10^{6} \text { cells }\right)^{-1}\right)$.

The hemozoin contents of three strains were compared. The three strains had been synchronized simultaneously and appeared by microscopy to be trophozoites of the same stage of maturation. Slightly more hemozoin was found in the chloroquine-resistant strains W2 and FCR3 (952 \pm 79 and $781 \pm 25$

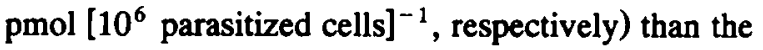
chloroquine-sensitive, $D_{6}$ strain $(637 \pm 91)$.

\subsection{Effects of antimalarials and protease inhibitors on hemozoin production and hypoxanthine uptake}

Hypoxanthine uptake and hemozoin production were measured in cultures containing synchronized small rings (Fig. 3A) or mature trophozoites (Fig. 3B), incubated in the presence of $0.5 \mu \mathrm{M}$ chloroquine, $0.5 \mu \mathrm{M}$ artemisinin, $50 \mu \mathrm{M}$ E64, or $50 \mu \mathrm{M}$ Pepstatin A. None of the compounds affected hypoxanthine uptake in rings. Hemozoin production by rings was inhibited by E64 (27\%) but was unaffected by the other compounds. The difference between the effect of E64 on hypoxanthine uptake and hemozoin content was statistically significant $(P=0.004)$. In contrast, all of the compounds caused substantial decreases in hypoxanthine uptake by trophozoites $(30-65 \%)$. E64 is the only compound that inhibited hemozoin production in trophozoites (37\%) more than hypoxanthine uptake (31\%), but this difference 
was not statistically significant. The other three compounds, chloroquine, artemisinin, and Pepstatin A, inhibited hemozoin production in trophozoites (10$30 \%$ ) to a much lesser extent than they inhibited hypoxanthine uptake $(60-65 \%)(P=0.002,0.0006$, and 0.0002 , respectively).

In order to further define the effects of E64 and chloroquine on hemozoin production, concentrationresponse curves were performed for hemozoin production and hypoxanthine uptake in both rings and trophozoites. At 2, 4, 20 and $100 \mathrm{nM}$, chloroquine inhibited hemozoin production in trophozoites significantly less than hypoxanthine uptake $(P=0.014$,
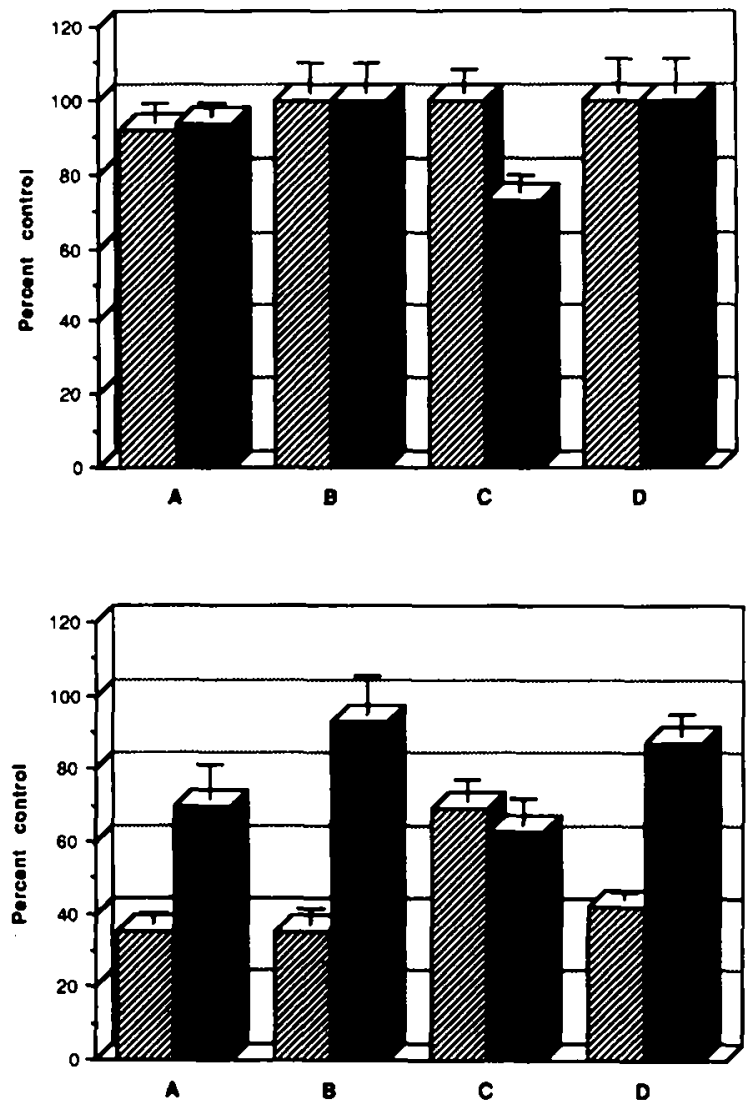

Fig. 3. Inhibition of hypoxanthine uptake (hatched bars) and hemozoin production (solid bars) in parasitized erythrocytes carrying rings (top) and trophozoites (bottom) by $0.5 \mu \mathrm{M}$ chloroquine (A), $0.5 \mu \mathrm{M}$ artemisinin (B), $50 \mu \mathrm{M}$ E64 (C), and $50 \mu \mathrm{M}$ pepstatin A (D). Triplicate cultures were incubated for $6 \mathrm{~h}$ and data are expressed as \% of control untreated cultures. Shown are means \pm standard deviations.
$0.0004,0.0001$, and 0.0003, respectively) (Fig. 4B). For rings (Fig. 4A), chloroquine inhibited hemozoin production less than hypoxanthine uptake at concentrations of 10,20 , and $100 \mathrm{nM}(P=0.0006,0.003$, and 0.002 , respectively). The differences in the effects of chloroquine on hemozoin production and hypoxanthine uptake were quite substantial at some concentrations. For example, at $10 \mathrm{nM}$, chloroquine inhibited ring hemozoin production by only $14 \%$ while it inhibited hypoxanthine uptake by $79 \%$. Similarly, trophozoite hemozoin production was only inhibited $34-37 \%$ at 20 and $100 \mathrm{nM}$ chloroquine while hypoxanthine uptake was inhibited by $79-80 \%$.

E64 had the opposite effect and inhibited parasite hemozoin production to a greater extent than hypoxanthine uptake for both rings (Fig. 4C) and trophozoites (Fig. 4D). The differences were statistically significant for trophozoites at $13,25,50$, and 100 $\mu \mathrm{M}(P=0.015,0.02,0.03$, and 0.04 , respectively) and for rings at 5 and $25 \mu \mathrm{M}(P=0.007$, and 0.014 , respectively). The effects are most marked in rings treated with $5 \mu \mathrm{M}$ and $25 \mu \mathrm{M}$ E64, where hemozoin production was inhibited by $33 \%$ and $46 \%$, while hypoxanthine uptake was inhibited by only $2 \%$ and $25 \%$, respectively.

\section{Discussion}

In this study, we have developed a rapid filtration assay for malarial hemozoin, and used it to study whether chloroquine, artemisinin, Pepstatin A, or E64 affect parasite hemozoin production. The effects of these compounds on hemozoin production were compared with their effects on hypoxanthine incorporation. Chloroquine, artemisinin and Pepstatin A, an aspartic protease inhibitor, affect hemozoin production to the same extent or less than hypoxanthine incorporation. In contrast, E64, a cysteine protease inhibitor, inhibits hemozoin production more than hypoxanthine uptake.

Malarial hemozoin consists of a large polymer, containing heme molecules linked to each other by bonds between ferric ions and carboxylate oxygens [25]. Because of the particulate nature of hemozoin, we used a $0.45-\mu \mathrm{m}$ filter to separate hemozoin heme from soluble hemoglobin-bound or free heme. The filters were found to retain $>96 \%$ of synthetic 
$\beta$-hematin but only $1 \%$ of unpolymerized heme (Table 1). Hemozoin isolated in this manner from infected red cells contained only about $1 \%$ hemoglobin-bound heme. Furthermore, uninfected red cells were found to contain $<2 \%$ as much filterable heme as ring-infected red cells. Thus, the assay is relatively specific for hemozoin (Table 2). Also, a linear correlation was found between parasitemia and hemozoin content (Fig. 1). Thus, the assay is sensitive enough to measure hemozoin in $100 \mu \mathrm{l}$ of packed red blood cells containing trophozoites at a $1 \%$ parasitemia (Fig. 1).

As added evidence for the validity of the filtration assay, a good correlation was found between this
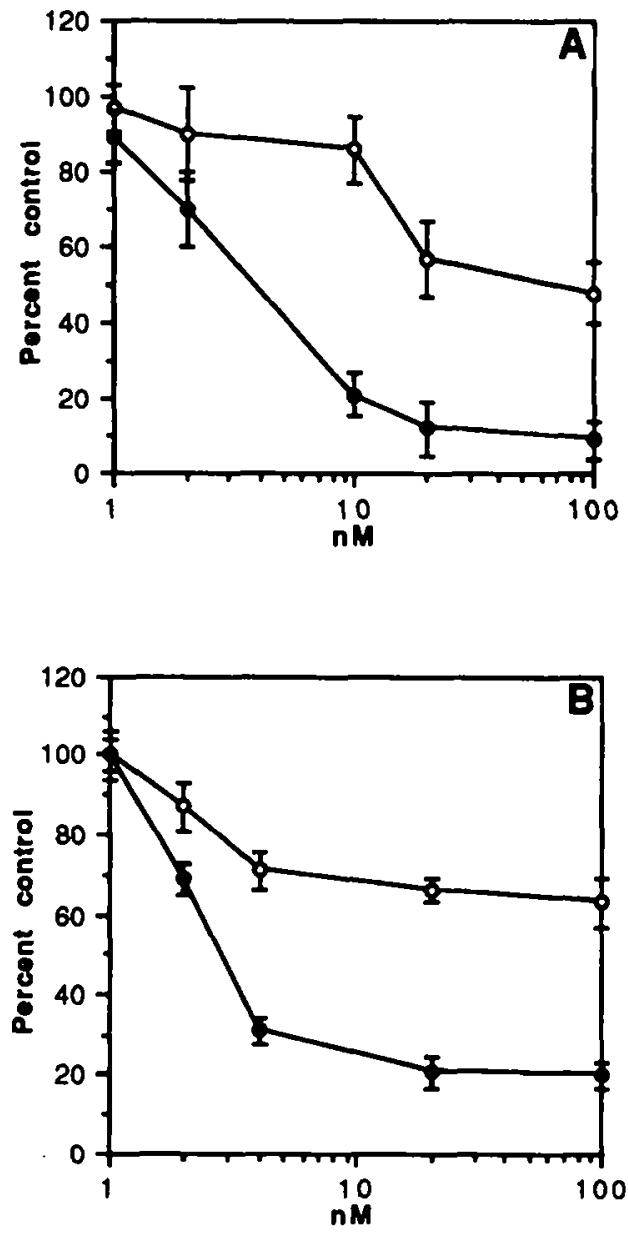

assay method and a previously published method (Fig. 2). Furthermore, the quantities of hemozoin reported here are similar to those reported by others. For example, the amounts of hemozoin reported by Orjih and Fitch [9] in rings (27-50 pmol [106 cells $]^{-1}$ ) and trophozoites (515-960 pmol [10 cells $]^{-1}$ ) are very comparable to those shown in Table 1. In addition, since the mean corpuscular hemoglobin of normal red cells is $29 \pm 2 \mathrm{pg}$ [29], $10^{6}$ red cells contain approximately $1800 \mathrm{pmol}$ of hemoglobin heme, and our data therefore suggest that the hemozoin of mature trophozoites contains $35-52 \%$ of the heme originally found in hemoglobin (Table 1). These data agree with previous estimates
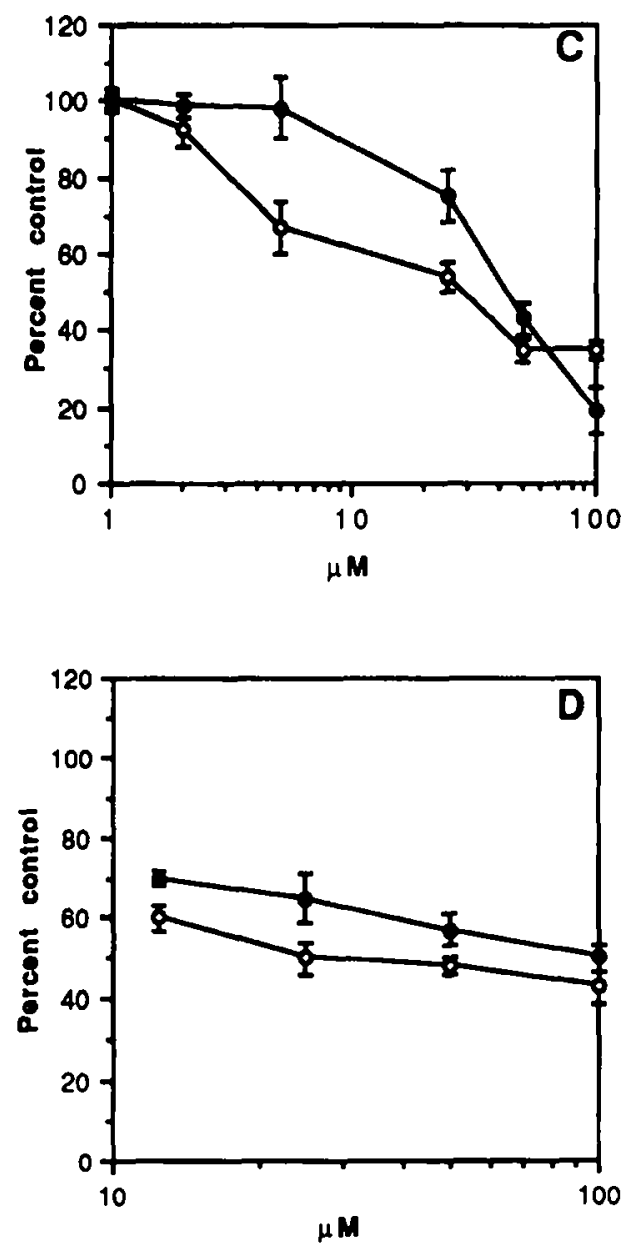

Fig. 4. Concentration-response curves of hemozoin production $(O)$ and hypoxanthine uptake $(O)$ in ring-infected erythrocytes by chloroquine (A) and E64 (C) and in trophozoite-infected erythrocytes by chloroquine (B) and E64 (D). Triplicate ring-infected and trophozoite-infected cultures were incubated $24 \mathrm{~h}$ and $6 \mathrm{~h}$, respectively. Shown are means \pm standard deviations. 
that $25 \%$ of hemoglobin in an infected cell is degraded [3].

The content of hemozoin in trophozoites is 8-fold higher than in rings (Table 2). This is not surprising since hemozoin is usually only observed by light microscopy in trophozoites. The hemozoin content of the ring stage might, in reality, be even lower, since the synchronized cultures of rings contained $2-5 \%$ trophozoites. However, it is also possible that hemoglobin degradation may have already started, since the ring stages used in this experiment represent $12-16 \mathrm{~h}$ of development.

The hemozoin content of the chloroquine-sensitive strain, $D_{6}$, at the mature trophozoite stage was slightly lower than that of the chloroquine-resistant strains (W2 and FCR-3) (Table 1). This is not consistent with the findings of Orjih and Fitch [9] who found that HB-3, a chloroquine-sensitive strain of $P$. falciparum, synthesized more $\beta$-hematin than Indochina I/CDC, a chloroquine-resistant strain. Thus it is likely that all of the differences are due to normal strain variation or to slight differences in the stages of development used. In aggregate, however, the data suggest that differences in hemozoin content between strains may not be associated with chloroquine resistance.

Chloroquine has been shown to have a number of biochemical effects [30-34], including the inhibition hemozoin biosynthesis in cell-free extracts [6]. Chloroquine-induced decreases of hemozoin content have recently been demonstrated in cell culture and in vivo $[9,10]$. But were these decreases in hemozoin content a direct result of heme polymerase inhibition or a consequence of parasite death due to another mechanism? In order to determine this, we set out to ascertain whether chloroquine affects hemozoin synthesis at concentrations at or below those which affect parasite viability.

Hypoxanthine incorporation was used here as an indicator of parasite viability. Hypoxanthine is taken up rapidly by malaria-infected red cells and incorporated into nucleic acid, predominantly RNA $[1,35]$. Hypoxanthine incorporation has been widely used to assess the effects of drugs on $P$. falciparum [27], and correlates well with morphological changes [28].

In this paper, the effects of chloroquine on hemozoin production were assayed at concentrations which were close to the drug's in vitro $50 \%$ inhibitory concentration $\left(\mathrm{IC}_{50}\right)$. As measured here, hypoxanthine uptake was inhibited by $50 \%$ at $3-6 \mathrm{nM}(1.5-3$ $\mathrm{ng} \mathrm{\textrm {ml } ^ { - 1 }}$ ) (Fig. 4A and $\mathrm{B}$ ) which is similar to the previously reported $\mathrm{IC}_{50}$ for $\mathrm{D}_{6}$ strain of $P$. falciparum $\left(2-7 \mathrm{ng} \mathrm{ml}^{-1}\right)$ [36]. Below the $\mathrm{IC}_{50}(1 \mathrm{nM})$ chloroquine has no effect on either hemozoin production nor hypoxanthine uptake in rings and trophozoites. At the $\mathrm{IC}_{50}$ or higher, hemozoin production is inhibited, but to a lesser extent than hypoxanthine incorporation.

These data are not inconsistent with the observations of Fitch and colleagues for both $P$. falciparum [9] and $P$. berghei $[10,11]$. Decreases in hemozoin production by $P$. falciparum in vitro were only observed by these investigators at chloroquine concentrations between 25 and $50 \mathrm{ng} \mathrm{ml}^{-1}$, which are very much higher than the reported $\mathrm{IC}_{50}$ values for chloroquine-sensitive $P$. falciparum $\left(2-7 \mathrm{ng} \mathrm{ml}^{-1}\right.$ ) [36]. Orjih and Fitch [9] reported no change in hemozoin production in cultures exposed to $12.5 \mathrm{ng}$ $\mathrm{ml}^{-1}(25 \mathrm{nM})$ chloroquine, which is closer to the $\mathrm{IC}_{50}$. Similarly, for $P$. berghei, diminished heme polymerase was only observed in mice treated with $62 \mathrm{mg} \mathrm{kg}^{-1}$ chloroquine which is over 12-fold higher than the reported $E_{9_{0}}$ for chloroquine in $P$. berghei-infected mice [37], which is far lower. For neither $P$. falciparum nor $P$. berghei did these investigators assess parasite viability other than by light microscopy which is relatively insensitive. Therefore, they could not determine whether the effect of the drug on hemozoin was greater or smaller than the effect of the drug on parasite viability.

Thus, chloroquine appears to affect hemozoin synthesis to a lesser extent than hypoxanthine uptake. This does not rule out the possibility that heme polymerase is still the target. The possibility exists that a small effect of chloroquine on hemozoin biosynthesis is sufficient to kill the parasite. The fact that chloroquine's effects on hemozoin content are only seen at high concentrations is also consistent with the possibility that the drug is killing parasites by an alternate mechanism and affecting hemozoin synthesis secondarily. Thus, there is still the need to consider alternate modes of action of this drug [3034].

The data presented here also have important implications for understanding the mode of action of artemisinin. We have recently proposed a two-step 
mechanism of action [16-18]: (1) heme-catalyzed activation of artemisinin into a free radical, and (2) alkylation of proteins and heme. However, we have hitherto been unable to determine whether protein alkylation or heme alkylation is more important. If heme alkylation were important in vivo, we postulated that artemisinin treatment might lead to a decrease in parasite hemozoin in either of two ways. First, alkylated hemes tend to be unstable and release iron [38]; artemisinin treatment might thus cause the degradation of hemozoin heme. Second, the artemisinin-heme adducts might act as an inhibitor of heme polymerase. However, the data presented here show that artemisinin treatment does not diminish hemozoin content and argue against, but do not rule out, the importance of heme as an alkylation target.

The effects of two protease inhibitors on hemozoin production were assessed. We initially used 50 $\mu \mathrm{M}$ of each, which is higher than the reported $\mathrm{IC}_{50} \mathrm{~S}$ (4 and $12 \mu \mathrm{M}$ for Pepstatin A and E64, respectively [39]). Pepstatin A, a specific aspartic protease inhibitor, did not inhibit hypoxanthine uptake or hemozoin production in ring-infected erythrocytes. For trophozoite-infected erythrocytes, Pepstatin A inhibited hemozoin production in trophozoites by only about $10 \%$, but hypoxanthine incorporation by $>60 \%$. This suggests that Pepstatin A may not directly inhibit hemozoin production. In contrast, E64, a specific cysteine protease inhibitor, inhibits hemozoin production more than hypoxanthine uptake in both rings and trophozoites at a variety of concentrations. These results imply that E64 directly affects hemozoin production, perhaps by inhibiting a cysteine protease involved in hemoglobin degradation [5]. These data are consistent with the observations of Rosenthal et al. [5], who found that the food vacuoles of E64-treated parasites stained more darkly than the food vacuoles of untreated parasites, suggesting the continued presence of undigested hemoglobin. The $P$. falciparum cysteine protease has recently been molecularly cloned [40], and may therefore offer a promising new target for antimalarial chemotherapy.

\section{Acknowledgements}

This work was supported by grants from the NIH (AI26848) and the World Bank/UNDP/World
Health Organization Special Programme for Research and Training in Tropical Diseases. We thank Renee Alli for technical assistance. We would also like to thank Drs. Philip Rosenthal and Hagai Ginsburg for helpful discussions.

\section{References}

[1] Sherman, I.W. (1979) Biochemistry of Plasmodium (malarial parasites). Microbiol. Rev. 43, 453-494.

[2] Slater, A.F. (1992) Malaria pigment. Exp. Parasitol. 74, 362-365.

[3] Roth, E.F., Jr., Brotman, D.S., Vanderberg, J.P. and Schulman, S. (1986) Malarial pigment-dependent error in the estimation of hemoglobin content in Plasmodium falciparum-infected red cells: Implication for metabolic and biochemical studies of the erythrocytic phases of malaria. Am. J. Trop. Med. Hyg. 35, 906-911.

[4] Glodberg, D.E., Slater, A.F.G., Cerami, A. and Henderson, G.B. (1990) Hemoglobin degradation in the malaria parasite Plasmodium falciparum: an ordered process in a unique organelle. Proc. Natl. Acad. Sci. USA 87, 2931-2935.

[5] Rosenthal, P.J., McKerrow, J.H., Aikawa, M., Nagasawa, H. and Leech, J.H. (1988) A malarial cysteine proteinase is necessary for hemoglobin degradation by Plasmodium falciparum. J Clin. Invest. 82, 1560-1566.

[6] Slater, A.F.G. and Cerami, A. (1992) Inhibition by chloroquine of a novel haem polymerase enzyme activity in malaria trophozoites. Nature 355, 167-169.

[7] Arese, P., Turrini, F. and Ginsburg, H. (1991) Erythrophagocytosis in malaria: Host defence or menace to the macrophage? Parasitol. Today 7, 25-28.

[8] Schwarzer, E., Turrini, F. Ulliers, D. Giribaldi, G. Ginsburg, H. and Arese, P. (1992) Impairment of macrophage functions after ingestion of Plasmodium falciparum-infected erythrocytes or isolated malarial pigment. J. Exp. Med. 176, 10331041.

[9] Orjih, A.U. and Fitch, C.D. (1993) Hemozoin production by Plasmodium falciparum: variation with strain and exposure to chloroquine. Biochim. Biophys. Acta 1157, 270-274.

[10] Chou, A.C. and Fitch, C.D. (1992) Heme polymerase: modulation by chloroquine treatment of a rodent malaria. Life Sci. 51, 2073-2078.

[11] Chou, A.C. and Fitch, C.D. (1993) Control of heme polymerase by chloroquine and other chloroquine derivatives. Biochem. Biophys. Res. Commun. 195, 422-427.

[12] Meshnick, S.R., Thomas, A., Ranz, A., Xu, C.M. and Pan, H.Z. (1991) Artemisinin (qinghaosu): the role of intracellular hemin in its mechanism of antimalarial action. Mol. Biochem. Parasitol. 49, 181-190.

[13] Zhang, F., Gosser, D. and Meshnick, S.R. (1992) Hemincatalyzed decomposition of artemisinin (qinghaosu). Biochem. Pharmacol. 43, 1805-1809. 
[14] Meshnick, S.R., Yang, Y.Z., Lima, V., Kuyers, F., Kamchonwongpaisan, S. and Yuthavong, Y. (1993) Iron-dependent free radical generation from the antimalarial agent artemisinin (qinghaosu). Antimicrob. Agent. Chemother. 37, 1108-1114.

[15] Posner G.H. and Oh, C.H. (1992) A regiospecifically oxygen-18 labeled 1, 2, 4-trioxane: a simple chemical model system to probe the mechanism(s) for the antimalarial activity of artemisinin (qinghaosu). J. Am. Chem. Soc. 114, 8328-8329.

[16] Yang, Y.Z., Asawamahasakda, W. and Meshnick, S.R. (1993) Alkylation of human albumin by the antimalarial artemisinin. Biochem. Pharmacol. 46, 336-339.

[17] Asawamahasakda, W., Benakis, A. and Meshnick, S.R. (1994) The interaction of artemisinin with red cell membranes. J. Clin. Lab. Med. 123, 757-762.

[18] Hong, Y-L, Yang, Y-Z and Meshnick, S.R. (1994) The interaction of artemisinin with malarial hemozoin. Mol. Biochem. Parasitol. 63, 121-128.

[19] Trager, W. and Jensen, J.B. (1976) Human malaria parasites in continuous culture. Science 193, 673-675.

[20] Lambros, C. and Vanderberg, J. P. (1979) Synchronization of Plasmodium falciparum erythrocytic stages in culture. J. Parasitol. 65, 418-420.

[21] Silamut, K. and White, N.J. (1993) Relation of the stage of parasite development in the peripheral blood to prognosis in severe falciparum malaria. Trans. R. Soc. Trop. Med. Hyg. 87, 436-443.

[22] Fairfield, A.S., Meshnick, S.R. and Eaton, J.W. (1983) Malaria parasites adopt host cell superoxide dismutase. Science 221, 764-766.

[23] Fuhrhop, J.H. and Smith, K.M. (1975) Laboratory methods. In: Porphyrins and Metalloporphyrins (Smith, KM. ed.), pp. 757-869. Elsevier, Amsterdam.

[24] Meshnick, S.R., Chang, K.P. and Cerami, A. (1977) Heme lysis of the bloodstream forms of Trypanosoma brucei. Biochem. Pharmacol. 26, 1923-1928.

[25] Slater, A.F.G., Swiggard, W.J., Orton, B.R., Flitter, W.D., Goldberg, D.E., Cerami, A. and Henderson, G.B. (1991) An iron-carboxylate bond links the heme units of malaria pigment. Proc. Natl. Acad. Sci. USA 88, 325-329.

[26] Drabkin, D.L. (1946) Spectrophotrometric studies. XIV. The crystallographic and optical properties of the hemoglobin of man in comparison with those of other species. J. Biol. Chem. 164, 703-723.
[27] Desjardins, R.E., Canfield, C.J., Haynes, J.D. and Chulay, J.D. (1979) Quantitative assessment of antimalarial activity in vitro by a semiautomatated microdilution techniques. Antimicrob. Agents Chemother. 16, 710-718.

[28] Chulay, J.D., Haynes, J.D. and Diggs, C.L. (1983) Plasmodium falciparum: Assessment of in vitro growth by $\left[{ }^{3} \mathrm{H}\right.$ ]hypoxanthine incorporation. Exp. Parasitol. 55, 138-146.

[29] Dacie, J.V. and Lewis, S.M. (1984) Practical Hematology, 6th edn. Churchill Livingstone, New York.

[30] Ginsburg, H. and Geary, T.G. (1987) Current concepts and new ideas on the mechanism of action of quinolone-containing antimalarials. Biochem. Pharmacol. 36, 1567-1576.

[31] Krogstad, D.J., Schlesinger, P.H. and Herwaldt, D.L. (1988) Antimalarial agents: mechanism of chloroquine resistance. Antimicrob. Agents. Chemother. 32, 799-801.

[32] Meshnick, S.R. (1990) Chloroquine as intercalator: a hypothesis revived. Parasitol. Today 6,77-79.

[33] Fitch, C.D. (1986) The mode of action of cholorquine and related malarial schizontocides. Parasitol. Today 2, 329-334

[34] Gabay, T., Krugliak, M., Shalmiev, G. and Ginsburg, H. (1994) Inhibition by anti-malarial drugs of haemoglobin denaturation and iron release in acidified red blood cell lysates: a possible mechanism of their anti-malarial effect? Parasitology $108,371-381$.

[35] Gutteridge, W.E. and Trigg, P.I. (1970) Incorporation of radioactive precursors into DNA and RNA of Plasmodium knowlesii in vitro. J. Protozool. 17, 89-96.

[36] Milhous, W.K., Gerena, L., Kyle, D.E. and Oduola, A.M.J. (1989) In vitro strategies for circumventing antimalarial drug resistance. Prog. Clin. Biol. Res. 313, 61-72.

[37] Peters, W. (1965) Drug resistance in Plasmodium berghei Vincke and Lips, 1948. III. Multiple drug resistance. Exp. Parasitol. 17, 97-102.

[38] Marks, G.S., McCluskey, S.A., Mackie, J.E., Riddick, D.S. and James, C.A. (1988) Disruption of hepatic heme biosynthesis after interaction of xenobiotics with cytochrome P-450. FASEB J. 2:2774-2783.

[39] Bally, E., Jambou, R., Savel, J. and Jaureguiberry, G. (1992) Plasmodium falciparum: differential sensitivity in vitro to E-64 (cysteine protease inhibitor) and Pepstatin A (aspartyl protease inhibitor). J. Protozool. 39, 593-599.

[40] Rosenthal, P.J. and Nelson, R.G. (1992) Isolation and characterization of a cysteine proteinase gene of Plasmodium falciparum. Mol. Biochem. Parasitol. 51, 143-152. 